\section{Daily defence mouthwash}

Corsodyl has introduced a daily maintenance mouthwash designed to help maintain healthy gums.

Formulated with a fresh mint taste, Corsodyl Daily Defence mouthwash contains low strength chlorhexidine digluconate and added fluoride to help protect against tooth decay.

The product is the only daily mouthrinse to contain the gold standard anti-plaque ingredient as well as fluoride.

Corsodyl, the UK's most popular mouthwash, also produces Mint and Original Mouthwash. All Corsodyl products have been proven to:

- treat and prevent chronic gingivitis

- inhibit the formation of dental plaque

- promote gingival healing

- maintain oral hygiene

- manage aphthous ulceration.

Corsodyl Dental Gel and Corsodyl Spray are also available. Samples can be requested from www.gskdentalprofessionals.co.uk.

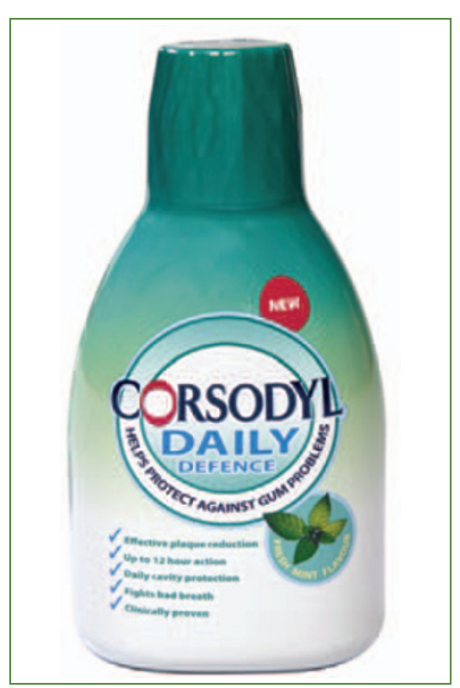

\section{Supremely clean}

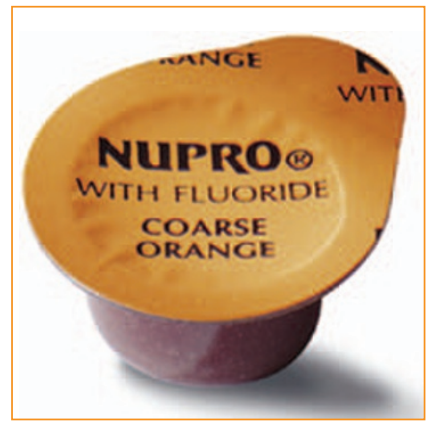

More and more patients expect a supreme 'clean' feeling after a hygiene appointment, which has led to an increase in the amount of prophylaxis being performed in UK dental surgeries.

The Prophy Paste Nupro currently holds the number one position in the UK market for delivering quality prophylaxis due to a combination of its low-

splatter formula and the range of options available:

- less splatter means less cleaning and a cleaner working environment

- it is available in tangy orange or fresh mint flavour, in both unit-dose cups (for better infection control) and in larger, multi-use tubs

- it is supplied in a range of three grit sizes: fine, medium and coarse.

Nupro provides DCPs with a range of cleaning and stain removing options.

For further details on Nupro or any other hygiene products, contact Dentsply on 01932853422.

\section{Instant ulcer relief}

With just one application of Oralmedic, mouth ulcer pain is eliminated.

Oralmedic provides a simple, hygienic method of treating mouth ulcers. In an easy-to-use cotton bud format, the patient simply snaps one end of the stick to permeate the bud and applies it to the affected area for 510 seconds.

With its active ingre-

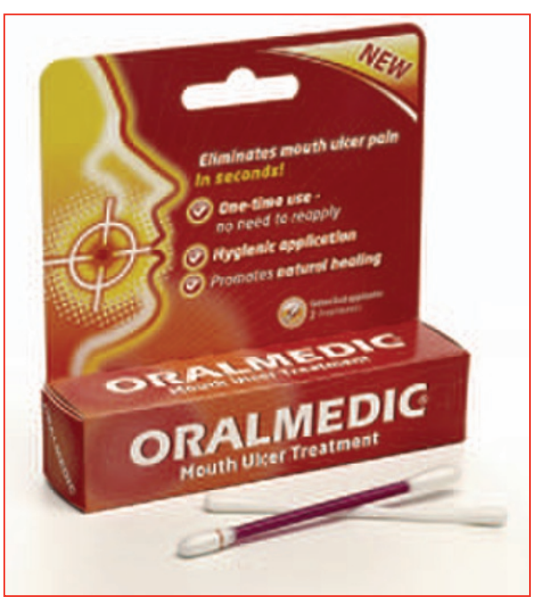
dient HybenX $\mathrm{X}^{\mathrm{TM}}$, Oralmedic gets straight to the infection site, seeks out dead and damaged tissue and reacts with it to create a seal over the site, thus protecting it and promoting the natural healing process.

'As many as $22 \%$ of the population suffer from mouth ulcers from time to time,' says Marketing Manager Nikki Pounds. 'Oralmedic is an ideal product for [DCPs] to recommend to patients suffering from this painful and uncomfortable condition.'

For more information telephone Ceuta Healthcare on 01202780558 or visit www.oralmedic.co.uk.

\section{Distraction by DVD}

An audiovisual cinema experience is now a reality in dentistry. The latest video eyewear is light and compact, and can be the ideal distraction for patients undergoing dental treatment.

'Facilitating and helping to get the patient's focus away from pain to pleasure pays off for both the patient and the (dental team),' said Dutch dentist Dr Rob Roef.

Many patients suffer during

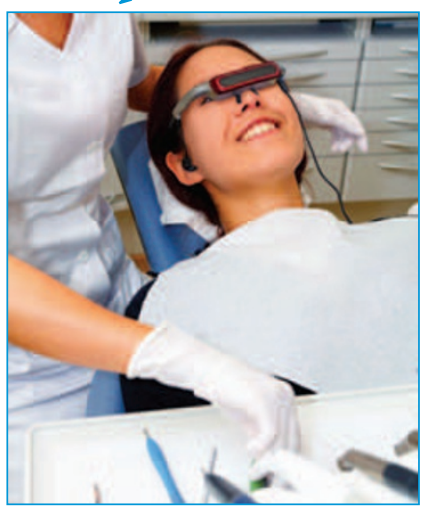
treatment simply because there is not much else to do other than focus on the pain, or anticipated pain. Distraction with video eyewear has been an option for several years, but the specifications have improved dramatically of late.

With the latest video eyewear the patient can still have eye contact with the dentist by focusing the eyes above the video screen. The volume level is adjustable and the option of one earphone only can be used. The eyewear can also be adjusted at the nose to provide optimum comfort and is smaller and less bulky than previous models, so that it does not present an obstacle to the dentist.

'The days of having something the size of a refrigerator on the patient's head are now gone,' added Dr Roef.

For further information see http://relaxview.eu. 